\title{
Las ideas fundamentales de probabilidad en el razonamiento de estudiantes de bachillerato
}

\author{
Ernesto Sánchez ${ }^{1}$, CINVESTAV-IPN (México) \\ Julio C. Valdez², CINVESTAV-IPN (México)
}

Recibido el 1 de Octubre de 2016; aceptado el 19 de Diciembre de 2016

\section{Las ideas fundamentales de probabilidad en el razonamiento de estudiantes de bachillerato}

\section{Resumen}

El objetivo de este trabajo es explorar las inferencias que los estudiantes de bachillerato formulan a partir de su conocimiento de las interpretaciones frecuencial y clásica de probabilidad. Se describen $y$ analizan los razonamientos de 30 estudiantes del $12^{\circ}$ grado, quienes cursaban la materia de Probabilidad y Estadística II. La recolección de datos se lleva a cabo mediante tres versiones de un cuestionario en los que se pide hacer predicciones y estimar probabilidades. El análisis de las respuestas revela la tendencia de los estudiantes al cálculo de probabilidades, mayormente apoyados en razonamientos inadecuados en los que intervienen las ideas de variabilidad, aleatoriedad $e$ independencia. Dichos razonamientos son descritos en una jerarquía con la finalidad de informar sobre las trayectorias de los estudiantes. Con base en este resultado, se sugiere que el primer objetivo en la enseñanza de la probabilidad debe ser el desarrollo de un razonamiento adecuado sobre estas ideas.

Palabras clave. Razonamiento probabilístico; Interpretación Clásica; Interpretación Frecuencial; Ley de los Grandes Números; Ideas fundamentales de probabilidad.

\section{Resumo}

O objetivo deste artigo é explorar as inferências feitas por estudantes do ensino médio através de seus conhecimentos sobre interpretações frequentista e clássica da probabilidade. O raciocínio de 30 alunos do $12^{\circ}$ ano que estavam estudando o assunto de Probabilidade e Estatística II é descrito e analisado. A coleta de dados foi através de três versões de um questionário, no qual se pediu para fazer previsões e avaliar probabilidades. A análise das respostas revela a tendência dos alunos para o cálculo de probabilidades, em sua maioria apoiados por insuficiente raciocínio em que estão envolvidas as idéias de variabilidade, aleatoriedade e independência. Tais argumentos são descritos em uma hierarquia, a fim de apresentar um relatório sobre o progresso dos alunos. Com base neste resultado, sugere-se que o primeiro objectivo do ensinamento de probabilidade deve ser o desenvolvimento de um raciocínio informal sobre estas ideias.

Palavras chave. Raciocínio probabilístico; Interpretação clásica; Interpretação frequentista; Lei dos grandes números; Idéias fundamentais da probabilidade.

\section{Abstract}

Para citar: Sánchez, E. \& Valdez, J.C. (2017). Las ideas fundamentales de probabilidad en el razonamiento de estudiantes de bachillerato. Avances de Investigación en Educación Matemática, 11, $127-143$. 
The aim of this paper is to explore the inferences made by high school students from their knowledge of the frequentist and classical interpretations of probability. The reasoning of 3012 th grade students who were studying the subject of Probability and Statistics II is described and analyzed. Data were collected through three versions of a questionnaire in which students were asked to make predictions and assess probabilities. The analysis of the responses reveals the students' tendency to the calculation of probabilities, mostly supported by inadequate reasoning in which the ideas of variability, randomness and independence are involved. This reasoning is described in a hierarchy for the purpose of reporting on the trajectories of the students. Based on this result, it is suggested that the first objective in the teaching of probability should be developing of an adequate reasoning about these ideas.

Key words. Probabilistic reasoning; Classical interpretation; Frequentist interpretation; Law of large numbers; Fundamental probability ideas.

\section{Résumé}

Le but de cet article est d'explorer les inférences faites par des élèves du secondaire sur la base de leur connaissance de les interprétations fréquentiste et classique de la probabilité. Le raisonnement de 30 élèves de 12 année qui étudiaient le sujet de la probabilité et de la statistique II est décrit et analysée. La collecte des données a été par le biais trois versions d'un questionnaire qui demande de faire des prévisions et d'évaluer les probabilités. L'analyse des réponses révèle la tendance des élèves per le calcul des probabilités, la plupart du temps pris en charge par un raisonnement inadéquat dans laquelle les idées de la variabilité, le hasard et l'indépendance sont impliqués. De tels arguments sont décrits dans une hiérarchie afin de faire rapport sur les progrès des élèves. Sur la base de ce résultat, il est suggéré que le premier objectif de l'enseignement des probabilités doit être le développement d'un raisonnement informel sur ces idées.

Paroles clés. Raisonnement probabiliste; Interprétation classique, Interprétation fréquentiste; Loi des grands nombres; Grandes idées de probabilité.

\section{Introducción}

Carnap (1945) señala que un problema importante en el desarrollo de la ciencia y las matemáticas es hacer más exacto un concepto ya en uso, o reemplazarlo por uno nuevo que sea más preciso. Este problema es una constante en el salón de clase, donde se busca que los estudiantes construyan sobre su conocimiento cotidiano conocimiento nuevo más técnico. En el caso de la probabilidad, si bien es cierto que los estudiantes de niveles básicos pueden entender algunas ideas probabilísticas de manera intuitiva, éstas no se suelen tener en cuenta en el diseño posterior de la enseñanza. Tampoco se suelen tener en cuenta en el diseño instruccional los sesgos y falsas concepciones que son comunes al resolver un problema sobre probabilidad $\mathrm{y}$, además, resultan resistentes al cambio, incluso después de la instrucción (Watkins, 1993). Usualmente, en la enseñanza se tiende a cubrir los contenidos y no tanto a proporcionar estrategias para superar las dificultades. De esta manera, se va generando una brecha entre el conocimiento cotidiano de los estudiantes y el conocimiento normativo que se les pide aprender. Por tanto, no es suficiente enseñar los procedimientos y conceptos en su forma abstracta, sino que es necesario organizarlos o reestructurarlos en un sistema articulado que considere las ideas previas de los estudiantes sobre probabilidad.

En México, la etapa del bachillerato es crucial para el aprendizaje de la probabilidad, pues es el periodo en el que las ideas previas de los estudiantes se enfrentan con el sistema de ideas formales. De este modo, resulta lícito tratar de entender cómo es el razonamiento probabilístico en este nivel, una vez que se ha recibido un curso de probabilidad. Infortunadamente, la probabilidad suele estudiarse como un conjunto de procedimientos de cálculo sin considerar que su aprendizaje implica un razonamiento nuevo, distinto al lógico-determinista que es característico de 
otras áreas de la matemática. Esto tiene como consecuencia que los estudiantes no utilicen de manera flexible los conocimientos adquiridos durante la enseñanza, por lo que sus concepciones, normalmente erróneas, se mantienen por encima del conocimiento normativo al tratar con situaciones que involucran incertidumbre. Así, el objetivo del presente trabajo es analizar el razonamiento probabilístico que despliega un grupo de estudiantes de bachillerato que tiene conocimiento sobre probabilidad, con el propósito de identificar aquellos aspectos de su conocimiento que desvían sus razonamientos, y contribuir, de esta forma, con información para un diseño de enseñanza en el que se consideren dichos aspectos.

\section{Antecedentes}

Un escenario promisorio para indagar sobre el razonamiento probabilístico de los estudiantes es la resolución de tareas que implican establecer relaciones entre las interpretaciones de probabilidad clásica y frecuencial. De acuerdo con Jones, Langrall y Mooney (2007), la investigación sobre estas interpretaciones proporciona un marco propicio para el estudio de las concepciones de los estudiantes acerca de las ideas clave en probabilidad. Al respecto, algunos estudios en los que se analiza cómo los estudiantes relacionan ambas interpretaciones (Ireland \& Watson, 2009; Prodromou, 2012), coinciden en que el conocimiento clave que subyace en esta relación, y que resulta difícil de entender para los estudiantes, es la Ley de los Grandes Números (LGN). Asimismo, muestran que entre los conceptos relacionados con esta ley se destacan las nociones de variabilidad, aleatoriedad e independencia, las mismas que se corresponden con lo que Gal (2005), en su trabajo sobre competencia probabilística, denomina las "grandes ideas" de probabilidad (en el sentido de fundamentales o básicas). En el presente estudio, asumimos que estas ideas son cruciales para desarrollar el razonamiento probabilístico. Un hecho que apoya este supuesto es la presencia de estudios sobre cada una de estas nociones; a continuación se mencionan algunos de ellos.

\subsection{Variabilidad}

La importancia de incluir el análisis de la variabilidad en los estudios de didáctica de la probabilidad y la estadística fue señalada inicialmente por Green (1993) y, más tarde, por Shaughnessy (1997), quien hizo un llamado a los educadores a investigar el pensamiento de los estudiantes sobre la variabilidad y su desarrollo. Desde entonces se ha estudiado el tema desde diferentes perspectivas. Por ejemplo, se han desarrollado jerarquías para describir la comprensión del concepto: prerrequisitos, reconocimiento, aplicaciones y aspectos críticos de la variación (Watson, Kelly, Callingham \& Shaughnessy, 2003). Por otro lado, la variabilidad se ha investigado con relación a problemas de predicción y muestreo. Al respecto, se ha descubierto que casi todos los estudiantes desde una edad temprana tienen una apreciación intuitiva de la variabilidad; sin embargo, carecen de la habilidad para decidir o evaluar qué variabilidad es razonable, además de no ser conscientes de la relación entre valor esperado y variabilidad (Watson \& Kelly, 2004). En particular, el presente trabajo se relaciona estrechamente con los estudios que analizan el razonamiento probabilístico acerca de las siguientes proposiciones: i) la variabilidad de las frecuencias relativas es grande cuando el número de repeticiones es pequeño; ii) pero es pequeña cuando el número de repeticiones es grande (Aspinwall \& Tarr, 2001; Stohl \& Tarr, 2002). 


\subsection{Aleatoriedad}

Entre los primeros trabajos en los que se aborda la comprensión de la aleatoriedad encontramos el de Piaget e Inhelder (1975) que plantearon que la comprensión de este concepto evoluciona en el individuo de acuerdo con las etapas del desarrollo cognitivo. No obstante, estudios posteriores como el de Metz (1998) contradicen este planteamiento. Otras investigaciones se han enfocado en la relación entre probabilidad y aleatoriedad que establecen tanto estudiantes como algunos profesionistas (Lecoutre, Rovira, Lecoutre \& Poitevineau, 2006), en las cuales se han identificado diversas concepciones sobre la aleatoriedad: como equiprobabilidad, como posibilidad, como incertidumbre, opuesta a la causalidad, derivada de un modelo y como una entidad que se puede presentar en distintos grados. Por otro lado, hay estudios en los que se examina la habilidad para generar e identificar secuencias aleatorias (Batanero, Arteaga, Serrano \& Ruíz, 2014; Batanero, Green \& Serrano, 1998; Sánchez, García \& Medina, 2015), los cuales coinciden en que las personas tienen dificultad para juzgar la aleatoriedad. El presente estudio se relaciona con investigaciones en las que se considera que la comprensión de la aleatoriedad implica el reconocimiento de la impredecibilidad de los resultados individuales y la regularidad estadística de estos sobre muchas repeticiones de la experiencia (Metz, 1998; Moore, 1990).

\subsection{Independencia}

Chernoff (2009) clasifica las tareas usadas en la investigación sobre percepción de la aleatoriedad en dos tipos: 1) tareas de predicción; y 2) tareas de reconocimiento. Estas tareas también permiten analizar la comprensión de la independencia (Batanero, 2016). Al respecto, se ha encontrado que los efectos de recencia positiva y negativa (Fischbein, 1975), la heurística de representatividad (Kahneman \& Tversky, 1972), y el enfoque al resultado aislado (Konold, 1989), son sesgos frecuentes en los razonamientos de los estudiantes (Konold, Pollatsek, Well, Lohmeier \& Lipson, 1993), que no favorecen la idea de independencia. Especialmente, Konold (1989) menciona la denominada falacia del jugador, o efecto de recencia negativa, como uno de los sesgos más comunes. Otras dificultades encontradas son la confusión de independencia de sucesos con sucesos excluyentes (Kelly \& Zwiers, 1986), la no diferenciación entre sucesos y experiencias independientes (Truran \& Truran, 1999) y la intervención del eje de tiempo (Falk, 1979). En general, se considera que dos eventos son independientes si la ocurrencia de uno de ellos no altera la probabilidad de ocurrencia (previa, simultánea o futura) del otro.

\section{Marco conceptual}

Jabareen (2009) define un marco conceptual como:

Una red, o un plano, de conceptos relacionados que proporcionan una manera de entender uno o varios fenómenos. Los conceptos que constituyen un marco se apoyan unos a otros, articulan sus respectivos fenómenos y establecen un marco filosófico específico. (p. 51)

Bajo esta definición, el marco que se utiliza en el presente trabajo se refiere al razonamiento probabilístico sobre las ideas fundamentales de probabilidad y está inspirado en el modelo más amplio de competencia probabilística definido por Gal (2005).

\subsection{Razonamiento probabilístico y las ideas fundamentales de probabilidad}


El razonamiento probabilístico aparece en alguna de las tres situaciones siguientes: 1) la solución de un problema de probabilidad y su justificación; 2) una cadena de argumentos para probar la verdad de una afirmación probabilística; y 3) el proceso mental que desarrolla un sujeto en 1) y 2). Conviene observar que tales razonamientos pueden distribuirse en diferentes niveles de rigor de acuerdo con las normas lógicas a las que se someten y a la elaboración simbólica con la que se formulan. Un razonamiento de un estudiante de primaria expresado con lenguaje ordinario y elaborado con una lógica básica puede no ser aceptable a un nivel de rigor más alto, por ejemplo, el de un estudiante de bachillerato que utiliza lenguaje simbólico. No obstante puede prefigurar un razonamiento válido. Un problema al estudiar el razonamiento probabilístico de los estudiantes es esclarecer el nivel de rigor con el cual juzgar sus producciones. En el presente estudio, se ha construido una jerarquía en la que se ubican los razonamientos de los estudiantes, del menos al más elaborado, ubicando en el nivel más alto los razonamientos con el rigor adecuado para examinar a los estudiantes del nivel de los participantes.

Por otro lado, es posible, en principio, dividir el conjunto de razonamientos probabilísticos en dos partes, aquellos en los que interviene alguna de las ideas de la probabilidad propuestas por Gal (2005): aleatoriedad, variación, independencia, predicción/Incertidumbre; y aquellos en los que no se presenta ninguna de estas ideas. Tradicionalmente se ha propuesto que los primeros se desarrollen preferentemente en el nivel universitario; no obstante, asumimos que tales ideas comparten lo que Heitele (1975) atribuía a las ideas fundamentales:

Cualquiera de estas ideas puede ser enseñada en forma comprensible a los niños de cualquier edad, siempre que se elija un lenguaje, grado de formalización y actividades adecuadas a su nivel de desarrollo cognitivo. (p. 187)

En este trabajo estamos interesados en enfocar la atención en la parte del razonamiento probabilístico de estudiantes de bachillerato en los que se presentan rasgos relacionados principalmente con la aleatoriedad, la variabilidad y la independencia. La intervención de estas ideas en un razonamiento puede darse a diferentes niveles de elaboración. Al respecto Gal (2005) aclara:

Algunos aspectos de estas grandes ideas pueden ser representados por símbolos matemáticos o términos estadísticos, pero su esencia no puede ser totalmente captada por notaciones técnicas. Los estudiantes deben entender sólo intuitivamente la naturaleza abstracta completa de estas ideas. (p. 46)

Debido a que nuestros sujetos son estudiantes de bachillerato que no habían recibido aún una instrucción enfocada sobre las ideas de aleatoriedad, variabilidad e independencia, se partió del supuesto de que mediante su esfuerzo al resolver las tareas que se les propusieran emergieran en sus razonamientos rasgos favorables o desfavorables a dichas ideas. La caracterización y análisis de tales rasgos puede contribuir en el diseño de secuencias de enseñanza para el desarrollo de un razonamiento probabilístico más completo. Para ello, es apropiado destacar aquellos aspectos de las mencionadas ideas que se deben tener en cuenta para contrastar los razonamientos que despliegan los estudiantes.

Variabilidad. De acuerdo con Moore (1990), "un objetivo de la instrucción en probabilidad debe ser ayudar a los estudiantes a entender que la variabilidad aleatoria, en lugar de la causalidad determinista, explica muchos aspectos del mundo" (p. 99). La variabilidad en probabilidad se refiere a las diferencias entre las frecuencias con que ocurren los eventos en una secuencia dada de experimentos y sus frecuencias 
esperadas, o las frecuencias relativas y la probabilidad de los eventos. Se puede referir también a la diferencia entre los valores de una variable aleatoria, o de densidad de probabilidad, o a la variación de los resultados en un experimento. En el presente estudio la variabilidad es vista a través de las siguientes proposiciones: la variabilidad de las frecuencias relativas es grande cuando el número de repeticiones de una experiencia aleatoria es pequeño, pero es pequeña cuando el número de repeticiones es grande.

Aleatoriedad. Actualmente, no se encuentra una definición simple que pueda ser utilizada de forma precisa para clasificar un evento o un proceso dado como aleatorio o no (Batanero, 2016). Por un lado, la aleatoriedad se asocia con la impredictibilidad de los resultados y con la regularidad estadística (Moore, 1990). Por otro lado, una secuencia de resultados se considera aleatoria si está desordenada y no tiene un patrón aparente, además de presentar rachas relativamente largas (Batanero \& Serrano, 1999). Formalmente, debe pasar ciertos test de aleatoriedad en los que las rachas serán más o menos largas dependiendo de la longitud de la secuencia en sí. En el presente estudio se opta por la primera acepción, pues parece ser más cercana al razonamiento de los estudiantes (Metz, 1998), y es más acorde con las tareas que se plantean. De esta manera, si se acepta que un fenómeno es aleatorio, se reconoce que sus resultados son impredecibles y se prevé la regularidad estadística sobre muchas repeticiones de la experiencia.

Independencia. La independencia es un concepto central de la probabilidad que se puede entender desde distintas perspectivas Por un lado, en un sentido intuitivo, existe lo que Truran y Truran (1999) denominan independencia de las experiencias. Por otro lado, existe independencia de sucesos dentro de un mismo experimento. La independencia se define formalmente mediante la regla del producto de probabilidades: los eventos $A_{1}, \ldots, A_{\mathrm{n}}$ son independientes si $P\left(A_{1} \cap \ldots \cap A_{\mathrm{n}}\right)=$ $P\left(A_{1}\right) \cdot \ldots \cdot P\left(A_{\mathrm{n}}\right)$. En el presente estudio se considera que la independencia tiene lugar cuando el resultado de un evento no altera las probabilidades de otros eventos (previos, simultáneos o futuros).

\section{Método}

Participantes. Los sujetos fueron 30 estudiantes de bachillerato del $12^{\circ}$ grado (22 mujeres y ocho hombres de entre 17 y18 años), quienes al momento de la recolección de datos se encontraban tomando el curso de Estadística y Probabilidad II; en específico, el tema de distribución binomial. De estos sólo un estudiante estaba repitiendo el curso. El grupo pertenecía a una institución pública de la Ciudad de México, cuya población total tomando el mismo curso era de 3,068 estudiantes.

Instrumento para la recolección de datos. La recolección de datos se realizó mediante tres versiones de un cuestionario, cada uno de los cuales fue respondido por 10 estudiantes. Además, se hicieron cuatro entrevistas, una por cada cuestionario, a excepción del Cuestionario I donde se entrevistó a dos estudiantes. Las situaciones que componen los cuestionarios son una adaptación que combina algunas tareas de comparación de probabilidades empleadas por Cañizares (1997) en un contexto de urnas, y las tareas de muestreo utilizadas en Metz (1998) y Stohl y Tarr (2002). A continuación se exponen las situaciones que integran el Cuestionario I, las respuestas normativas y las ideas que exploran. Se elige este cuestionario porque fue en el que se expusieron la mayor variedad de razonamientos. 
Situación 1. El resultado de 1,000 extracciones hechas de una urna que contiene cuatro bolas en total (entre blancas y negras), fue de 489 bolas blancas y 511 negras: a) ¿Cuántas bolas blancas y cuántas negras contiene la urna? b) Si se realiza la extracción 1,001, ¿qué color de bola crees que se obtendría? c) ¿Qué color de bola consideras que se obtuvo en la primera extracción?

La respuesta a la pregunta la es que la urna contiene dos bolas blancas y dos negras. El estudiante debe evaluar que la variabilidad de los resultados respecto a los valores del modelo equiprobable es poca y, por tanto, aceptable; el resultado sería casi imposible con cualquier otro modelo de urna con la restricción de contener cuatro bolas. En las respuestas a las preguntas $1 \mathrm{~b}$ y $1 \mathrm{c}$ (se puede presentar cualquier color de bola) se debe considerar el modelo establecido y la independencia de las extracciones; pero también se explora la aleatoriedad, en el sentido de que el estudiante debe inferir que no es posible predecir con certeza lo que se obtendrá en un ensayo, o decir lo que ocurrió en el primer ensayo.

Situación 2. Se tienen dos urnas: La urna B contiene seis bolas (blancas y negras) y la urna $\mathrm{C}$ contiene tres. Se hicieron 1,000 extracciones al azar de cada urna. De la urna $\mathrm{B}$ se obtuvieron 324 bolas blancas y 676 negras. De C se obtuvieron 344 blancas y 656 negras. a) ¿Cuál urna elegirías para hacer la extracción 1,001, de tal forma que la bola resultante sea negra? b) ¿Qué valor [numérico] le asignarías a que ocurra el evento 'Sacar una bola negra de la urna B en la extracción 1,001'? c) ¿Qué valor [numérico] le asignarías a que ocurra el evento 'Sacar una bola negra de la urna $\mathrm{C}$ en la extracción 1,001'?

En esta situación se espera que los estudiantes deduzcan los contenidos de ambas urnas: $\mathrm{B}$ con dos bolas blancas y cuatro negras; $\mathrm{C}$ con una blanca y dos negras. Una vez establecida esta hipótesis, la respuesta a la pregunta 2a (se puede elegir cualquier urna) se basa en la identificación de que ambas urnas son equivalentes, asumiendo que la diferencia entre los resultados y los valores esperados es parte de la variabilidad natural del fenómeno, ya que nueve y 11 de mil es relativamente poco. Se debe tener en cuenta también la independencia del resultado 'bola negra' de lo ocurrido en ensayos anteriores. Asimismo, es necesario considerar la aleatoriedad, es decir, que con ninguna urna se puede asegurar obtener bola negra en la extracción 1001. En las respuestas a las preguntas $2 \mathrm{~b}$ y $2 \mathrm{c}$ se deben considerar los modelos establecidos y la independencia de las extracciones, por lo que se espera que los estudiantes proporcionen la probabilidad $2 / 3$ en ambas preguntas. Aquí conviene aclarar que la razón por la que se pide asignar un valor numérico y no se solicita directamente una probabilidad fue con el propósito de destacar el carácter conceptual de la probabilidad (como una medida de la posibilidad que tiene un suceso o evento de ocurrir) y no sólo el procedimental.

Situación 3. Los resultados de sacar 10 bolas de cada una de las urnas B y C de la actividad anterior fueron: dos bolas blancas y ocho negras de la urna B, y cinco blancas y cinco negras de C. a) ¿Cuál urna elegirías para hacer la onceava extracción de tal forma que la bola resultante sea blanca? b) ¿Qué valor [numérico] le asignarías a que ocurra el evento 'Sacar una bola blanca de la urna B en la onceava extracción'? c) ¿Qué valor [numérico] le asignarías a que ocurra el evento 'Sacar una bola blanca de la urna $\mathrm{C}$ en la onceava extracción'?

En esta situación se espera que la respuesta a la pregunta 3 a sea que se puede elegir cualquier urna. La respuesta a las preguntas 3 b y $3 \mathrm{c}$ es $1 / 3$ en ambos casos. Las preguntas se responden bajo la hipótesis de que las extracciones son independientes y teniendo en cuenta el modelo. Sin embargo, puede surgir la cuestión de si la variabilidad de los resultados en la muestra, respecto al modelo, es aceptable. 
Teniendo en cuenta la aleatoriedad se puede deducir que una desviación de ocurrencias de dos o tres resultados respecto al valor esperado es posible. El valor esperado está entre seis y siete (6.67), luego los resultados ocho y cinco no representan desviaciones mayores a dos unidades.

Con los Cuestionarios II y III se busca observar el efecto que tiene en las respuestas de los estudiantes la modificación de algunas variables de las tareas, para tener una imagen más amplia de sus razonamientos. Así, el Cuestionario II se compone por las tres situaciones que conforman el Cuestionario I, con la diferencia de que el contenido de las urnas es conocido. Además, en cada situación se agregó una pregunta en la que se pide hacer predicciones sobre los resultados de 1,000 (1a y 2a) y 10 extracciones (3a). De esta manera, en la Situación 1 se presenta la urna A, la cual contiene dos bolas blancas y dos negras; y las Situaciones 2 y 3 tratan sobre las urnas B y C, cuyo contenido es de dos bolas blancas y cuatro negras, y una blanca y dos negras, respectivamente. Por su parte, en el Cuestionario III sólo se consideran estas dos últimas situaciones, pero, en esta ocasión, la distribución del contenido de cada una de las urnas $\mathrm{B}$ y $\mathrm{C}$ es desconocido, así como su total. En la Situación 2, únicamente se conoce la distribución de los resultados de dos muestras de 1,000 extracciones, tomadas una de cada urna (324 bolas blancas y 676 negras de la urna B; 344 blancas y 356 negras de la urna C); mientras que en la Situación 3 sólo se tiene información sobre dos muestras de 10 extracciones (dos blancas y ocho negras de la urna B; cinco blancas y cinco negras de la urna $\mathrm{C}$ ).

Procedimiento de ejecución. La aplicación de los cuestionarios se efectuó dentro del horario de clases y los estudiantes tuvieron 50 minutos para responderlo. Se les comunicó que los resultados contribuirían a su evaluación, con el fin de que se comprometieran con los problemas y sus soluciones. Las respuestas que proporcionaron fueron agrupadas para su análisis y clasificación.

\section{Análisis de resultados}

A partir de las respuestas de los estudiantes a las preguntas de los cuestionarios, ha sido posible construir una jerarquía que da cuenta de cuatro niveles de desarrollo de su razonamiento probabilístico; comenzando por la ausencia de éste en el nivel más bajo (Nivel 1), y terminando en su dominio, en el contexto de las preguntas, en el nivel más alto (Nivel 4). En la Tabla 1 se resumen las características generales que se lograron observar en las respuestas de los estudiantes respecto a la Variabilidad, Aleatoriedad e Independencia. A continuación se extienden un poco más tales descripciones. Los ejemplos que se proporcionan se refieren en su mayoría al Cuestionario I.

\subsection{Variabilidad}

En el Nivel 1 los razonamientos de los estudiantes se caracterizan por no aludir a la variabilidad. Por ejemplo, en la pregunta 1a del Cuestionario II se pide inferir el número de bolas blancas y de bolas negras que se obtendrían en 1,000 extracciones de la urna $\mathrm{A}$. La respuesta normativa es que se podría obtener un número de bolas blancas o de bolas negras entre $500 \pm 50$ con una probabilidad de 0.997 . Una respuesta en la que se aprecia la ausencia de variabilidad es la que presenta A13, en cuya respuesta tal parece que conocer las probabilidades implicadas propicia que el estudiante dé la respuesta teóricamente esperada, omitiendo la variabilidad (Shaughnessy, Watson, Moritz \& Reading, 1999).

Como hay 2 blancas y dos negras cada color tiene $1 / 2$ de probabilidad de salir, por lo 
tanto, son equiprobables, y entonces si multiplicamos 1,000 por la probabilidad de que salga negra o blanca nos dará 500 (A13).

Los razonamientos en el Nivel 2 tienen en cuenta la variabilidad, pero se hace un tratamiento inadecuado de ella. En la pregunta 1a del Cuestionario I la respuesta normativa es que el contenido de la urna es de dos bolas blancas y dos negras, ya que bajo esta hipótesis el resultado dado de las 1,000 extracciones es más plausible que con cualquier otro modelo de urna. Una respuesta en la que no se hace un tratamiento adecuado de la variabilidad es la que proporciona A1, quien juzga que la pequeña diferencia entre la frecuencia empírica y la esperada es significativa.

Con los resultados dados [511 bolas negras y 489 blancas], se estimaría que hay un número mayor de bolas negras [en la urna $A$ ], ya que su probabilidad es más alta $(511 / 1000)$, mientras que de la blanca es lo contrario $(489 / 1000)$ ( $\Rightarrow$ yo diría que hay 3 bolas negras y una bola blanca) a notar por su probabilidad (A1).

En este nivel también se incluyen los razonamientos de los estudiantes que infieren que una muestra pequeña también es representativa de la población de origen. Esto es, que los resultados de 10 extracciones son suficientes para inferir el contenido de la urna.

Tabla 1. Niveles de razonamiento con relación a las ideas fundamentales de probabilidad.

$\begin{array}{llll}\text { Nivel } 1 & \text { Nivel } 2 & \text { Nivel } 3 & \text { Nivel } 4\end{array}$

\begin{tabular}{|c|c|c|c|c|}
\hline & $\begin{array}{l}\text { No consideran la } \\
\text { variabilidad. }\end{array}$ & $\begin{array}{l}\text { Tanto en muestras } \\
\text { pequeñas como en } \\
\text { grandes creen que las } \\
\text { diferencias entre las } \\
\text { frecuencias empíricas y } \\
\text { las frecuencias } \\
\text { esperadas son } \\
\text { significativas. }\end{array}$ & $\begin{array}{l}\text { Estiman que en una } \\
\text { muestra grande las } \\
\text { diferencias no son } \\
\text { significativas, pero } \\
\text { juzgan que para una } \\
\text { muestra pequeña que } \\
\text { procede de la misma } \\
\text { población la diferencia } \\
\text { que se presenta tampoco } \\
\text { puede ser significativa. }\end{array}$ & $\begin{array}{l}\text { Reconocen que la } \\
\text { variabilidad es grande } \\
\text { cuando la muestra es } \\
\text { pequeña, pero es poca } \\
\text { cuando la muestra es } \\
\text { grande (Observan el } \\
\text { papel del tamaño de la } \\
\text { muestra). }\end{array}$ \\
\hline & $\begin{array}{l}\text { Hacen una } \\
\text { predicción } \\
\text { determinista; } \\
\text { Infieren una } \\
\text { regularidad } \\
\text { aparente, } \\
\text { apoyados en una } \\
\text { sola muestra. }\end{array}$ & $\begin{array}{l}\text { Hacen una predicción } \\
\text { determinista } \\
\text { matizándola con } \\
\text { lenguaje probabilístico } \\
\text { Distinguen } \\
\text { regularidades } \\
\text { circunstanciales. }\end{array}$ & $\begin{array}{l}\text { Reconocen que no se } \\
\text { puede predecir el } \\
\text { resultado con exactitud, } \\
\text {; pero hay una inclinación } \\
\text { por un resultado } \\
\text { particular; Distinguen } \\
\text { regularidades } \\
\text { acompañadas de sesgos. }\end{array}$ & $\begin{array}{l}\text { Reconocen que no se } \\
\text { puede predecir con } \\
\text { certeza el resultado de } \\
\text { lun ensayo, pero sí la } \\
\text { estabilidad de las } \\
\text { frecuencias alrededor } \\
\text { de un valor } \\
\text { (probabilidad) en el } \\
\text { largo plazo. }\end{array}$ \\
\hline & $\begin{array}{l}\text { Consideran que } \\
\text { resultados } \\
\text { previos o futuros } \\
\text { influyen en el } \\
\text { posible resultado } \\
\text { de un ensayo. }\end{array}$ & $\begin{array}{l}\text { Creen que el posible } \\
\text { resultado de un ensayo } \\
\text { depende de qué tan } \\
\text { representativa es la } \\
\text { muestra de la } \\
\text { población. }\end{array}$ & $\begin{array}{l}\text { Se valen del modelo } \\
\text { para determinar el } \\
\text { posible resultado de un } \\
\text { ensayo. }\end{array}$ & $\begin{array}{l}\text { Reconocen la } \\
\text { independencia, y } \\
\text { utilizan el modelo para } \\
\text { inferir el posible } \\
\text { resultado de un } \\
\text { ensayo. }\end{array}$ \\
\hline
\end{tabular}


En el Nivel 3 los razonamientos se caracterizan por hacer un tratamiento parcialmente adecuado de la variabilidad. Por ejemplo, continuando con la pregunta 1a del Cuestionario I, una respuesta que se ubica en este nivel es la que da A10:

La distribución de las bolas [en la urna $A$ ] es equitativa, es decir, hay dos bolas blancas y dos negras, porque se ve en los resultados obtenidos [511 bolas negras y 489 blancas]. No hay mucha diferencia más que de 22 extracciones, por lo que tienen la misma probabilidad de extracción (A10).

Sin embargo, para una muestra pequeña que procede de la misma población se juzga que la diferencia debería seguir siendo pequeña $\mathrm{y}$, por tanto, no significativa. Esto se aprecia en el razonamiento de A7 en la pregunta 3a del Cuestionario I durante la entrevista. En este nivel, tal parece que los estudiantes toman como premisa una creencia, en la cual infieren que a mayor probabilidad de obtener una bola negra, respecto la probabilidad de extraer una bola blanca, mayor la cantidad de bolas negras, sobre las blancas, en muestras de cualquier tamaño.

$\mathrm{Si}$ vemos la probabilidad es una de tres [de obtener una bola blanca de la urna $C$ ] entonces es para que los resultados [en la muestra] no estén tan iguales... son cinco bolas blancas y cinco bolas negras, pero como son tres [bolas en la urna $C$ ] no puede haber una bola y media blanca, y una bola y media negra (A7).

En el Nivel 4 se exhibe un razonamiento consolidado sobre variabilidad. Por ejemplo, siguiendo con la pregunta 1a, además de estimar que de las cuatro bolas que contiene la urna A dos son blancas y dos negras, aunque que la pequeña diferencia entre la frecuencia empírica y la esperada no es significativa en una muestra grande, se juzga que en una muestra pequeña que procede de la misma población dicha diferencia puede ser significativa. A partir de esto último, se determina que una muestra pequeña no es representativa de la población de origen. En otros términos, se aprecia la relación entre el tamaño de la muestra y la variabilidad de los resultados.

\subsection{Aleatoriedad}

En el Nivel 1 los razonamientos de los estudiantes son deterministas. Esto se refiere a la ausencia de expresiones que indiquen incertidumbre acerca de lo que puede ocurrir en un ensayo. Por ejemplo, en la pregunta $1 \mathrm{~b}$ del Cuestionario I la respuesta normativa es que se podría obtener cualquier color de bola en la extracción 1,001, debido a que, con la información dada, el modelo de urna más plausible es el que contiene dos bolas blancas y dos negras. Una respuesta en la que no se aprecia incertidumbre sobre lo que puede ocurrir en la extracción es la que proporciona A. El alumno no considera que la mayor frecuencia de bolas negras no garantiza como resultado una bola de este color en la siguiente extracción. En este nivel también se ubican los razonamientos en los que se infiere una regularidad de las frecuencias a partir de una sola muestra.

En la extracción 1,001 se obtendría una bola negra, ya que tienen mayor cantidad de bolas negras, según muestra la tabla 1 [489 bolas blancas y 511 negras (A4)”.

En los razonamientos de Nivel 2 se utiliza lenguaje probabilístico, pero las respuestas siguen siendo deterministas. Por ejemplo, continuando con la pregunta $1 \mathrm{~b}$, una respuesta característica de este nivel es la que da A1:

Se estimaría que salga negra [en la extracción 1,001], ya que su probabilidad es mayor. Negra (511/1000) (A1).

Aquí es necesario tener en cuenta que la probabilidad se ve como un atributo de la muestra, de modo que en cada repetición de le experiencia habría una probabilidad 
distinta. En este nivel también están los razonamientos en los que se emplean expresiones como "puede ser que...", "creo que...", etc., que indican una percepción de la no predictibilidad del resultado. Sobre las regularidades que se observan, estas son circunstanciales. Esto es, una vez hecha la simulación de la experiencia puede ocurrir que se sigan obteniendo más bolas negras que blancas, lo que puede ser un indicio para los estudiantes de que esto es una tendencia que se mantiene.

En el Nivel 3 los razonamientos se distinguen por reconocer que no es posible saber con precisión el resultado de un ensayo, pero hay una inclinación por uno en particular. Siguiendo con la pregunta $1 \mathrm{~b}$, una respuesta que ejemplifica este razonamiento es la que presenta A10:

Posiblemente la blanca [se obtendría en la extracción 1,001], porque tienen la misma probabilidad que la negra (A10).

Una explicación plausible de este tipo de razonamiento es que para los estudiantes expresiones como "Se puede obtener cualquier color de bola" no son vistas como respuestas adecuadas debido a su carácter incierto. Aquí también se encuentran los razonamientos en los que se considera que, sobre muchas repeticiones de la experiencia, la frecuencia relativa de cada resultado se acerca a la probabilidad del evento correspondiente, pero se piensa que la convergencia es exacta.

En los razonamientos de Nivel 4, se reconoce que no es posible predecir con certeza el resultado de un ensayo, por lo que respuestas indeterminadas son vistas como válidas. Por ejemplo, continuando con la pregunta $1 \mathrm{~b}$, la respuesta apropiada es que se puede obtener cualquier color de bola en la extracción 1,001 , ya que la probabilidad no favorece a un resultado en particular. Esto bajo el supuesto de que la urna A contiene dos bolas blancas y dos negras. Además, se identifica que, sobre muchas repeticiones de la experiencia, la las frecuencias relativas de los posibles resultados se estabilizan alrededor de las probabilidades teóricas correspondientes.

\subsection{Independencia}

En el Nivel 1 los razonamientos de los estudiantes se corresponden con un tipo de recencia positiva o negativa. Por ejemplo, en la pregunta 1c del Cuestionario I, bajo el supuesto de que en la urna A hay dos bolas blancas y dos negras, la respuesta normativa es que pudo haber resultado cualquier color de bola en la primera extracción. Una respuesta que se apoya únicamente en los resultados de la muestra es la que expone A5 donde el estudiante considera que la información de la muestra es relevante para responder lo que ocurrió en la primera extracción, y aplica una especie de razonamiento bayesiano: "Si en la muestra salieron más negras, es más probable que en la primera extracción haya salido negra".

Negra, puesto que si hubo más resultados de extraer bola negra [511 bolas negras $y$ 489 blancas], puede ser que en las primeras [extracciones] haya resultado en su mayoría la [bola] negra (A5). [En la primera extracción se obtuvo una bola].

Los razonamientos de Nivel 2, además de apoyarse en los resultados de la muestra, también consideran qué tan representativos son estos de la población. Por ejemplo, en la Situación 1 del Cuestionario I, bajo las hipótesis de que la urna A contiene dos bolas blancas y dos negras, y que después de 10 extracciones se obtendrían siete bolas blancas y tres negras, A7 señala que resultaría una bola negra en la siguiente extracción. Sin embargo, la respuesta normativa es que se puede obtener cualquier color de bola, pues la probabilidad de obtener una bola blanca es igual a la probabilidad de extraer una negra. 
Yo creo que, para balancear un poco más esto [siete bolas blancas y tres negras], yo creo que sacaría como una bola negra [en la onceava extracción] (A7).

En el Nivel 3 los razonamientos de los estudiantes se distinguen por estar basados en el modelo, pero sin hacer alusión a la nula influencia que tienen los resultados previos (o futuros) sobre el resultado de un ensayo. Siguiendo con la pregunta 1c, la respuesta que ejemplifica este nivel es la normativa tal como lo expresa A6. Sin embargo, no hay un reconocimiento explícito de la independencia del resultado respecto de las 1,000 extracciones acontecidas. En cambio, en el Nivel 4 es el supuesto de independencia lo que permite ignorar lo ocurrido y razonar a partir del modelo para determinar que en la primera extracción se puedo haber obtenido cualquier color de bola.

Si tenemos 2 bolas negras y 2 bolas blancas las probabilidades son $1 / 2$ y $1 / 2$. Por ende, pudo ser cualquier color de bola la primera extracción (A6).

\subsection{Concepción de probabilidad}

A pesar de que algunos estudiantes expusieron razonamientos apropiados sobre las ideas de variabilidad, aleatoriedad e independencia, su concepción poco consolidada de probabilidad desvió sus razonamientos de las respuestas normativas. Por un lado, están los razonamientos en los que se incurre en alguno de los sesgos de atención. Por ejemplo, en la pregunta 2 a del Cuestionario I la respuesta normativa es que se puede elegir cualquier urna para hacer la extracción 1,001 de tal forma que se obtenga una bola negra, ya que con la información dada, los modelos de urnas más plausibles son equivalentes: urna $\mathrm{B}$ con dos bolas blancas y cuatro negras, y urna $\mathrm{C}$ con una blanca $\mathrm{y}$ dos negras. Una respuesta en la que se observa un razonamiento restringido que se enfoca en el número de elementos favorables al evento objetivo es la que presenta A10

Para hacer la extracción 1,001 elegiría la $\mathrm{B}$, porque, aunque básicamente [ambas urnas] tienen la misma probabilidad [de dar como resultado una bola negra], se refleja que hay más posibilidad de obtener bola negra en la urna $\mathrm{B}$ [ya que contiene más bolas negras].

Una posible explicación de este tipo de conducta es que los estudiantes no consideran aceptables respuestas como "de cualquier urna se puede extraer una bola negra". De esta manera, ignoran la igualdad entre las probabilidades y buscan algún otro elemento que les permita dar una respuesta 'precisa'. Esto los lleva a considerar que el resultado de un ensayo depende de una característica particular del generador aleatorio. En este caso, de la mayor cantidad de bolas negras en las urnas. Sin embargo, se abre la interrogante del significado que los estudiantes le dan a la probabilidad. En específico, de la relación que guarda con la incertidumbre. Al respecto, como parte de una de las entrevistas, se le pregunta a A7 acerca de cuál consideraba que es la relación entre la suerte, o el azar, con la probabilidad. El estudiante juzga que sólo la probabilidad 50-50 tiene relación con el azar, en el sentido de que expresa el mismo significado. Esto es, que cualquier cosa se puede presentar:

El azar, si lo vemos en probabilidad, es el 50\% de éxito y el 50\% de fracaso, ya que no sabes, no te inclinas ni a un lado ni al otro, estás en el punto medio (A7).

Sin embargo, continuando con la entrevista, el estudiante no articula de forma apropiada la probabilidad con la incertidumbre, pues para cualesquier otros valores de probabilidad no vislumbra esta relación, lo que, al parecer, propicia que incurra en el sesgo del resultado aislado (Konold, 1989):

En la probabilidad mínimo sabes que tienes un porcentaje de éxito, o ya sea un 
porcentaje de fracaso [...]. O sea, la probabilidad sí mínimo sabes si fracasas o tienes un éxito. $\mathrm{Y}$ en el azar no. En el azar estás como que en el punto medio, porque no sabes si pierdes o ganas (A7).

Por tanto, el problema de no distinguir que la incertidumbre se mantiene, sólo que en distinto grado, radica en que, para algunos estudiantes, el conocer la probabilidad de que un evento ocurra implica la exclusión de la incertidumbre (Konold et al., 2011). Por su parte, a los otros tres estudiantes entrevistados también se les cuestionó acerca de la relación que creen existe entre probabilidad e incertidumbre, o el azar. A4 se refiere a la probabilidad como un valor que se asocia a un evento binomial, pero no menciona a la incertidumbre; A13 considera que hay una relación estrecha entre estos dos conceptos, pero no logra especificarla de manera clara; y A29, aunque no expresa un razonamiento cuantitativo, menciona a la probabilidad como una forma de tratar con el azar.

\section{Discusión y Conclusiones}

Las inferencias, representaciones e interpetaciones que los estudiantes realizan ante situaciones probabilistas que involucran las definición clásica y el enfoque frecuencial de probabilidad se ven influidas por el nivel de razonamiento que hayan adquirido acerca de, y con, las ideas de variabilidad, aleatoriedad e independencia. En el análisis de las respuestas se ha reflejado que tales ideas "interactúan cada una con otra en formas complejas durante las conductas de los estudiantes o durante su aprendizaje" (Gal, 2005, p. 45); así es frecuente que ciertas respuestas se puedan explicar o predecir por el nivel de razonamiento del estudiante sobre aleatoriedad, pero también por su nivel de razonamiento sobre variabilidad o sobre independencia.

Los niveles de razonamiento sobre variabilidad que se han caracterizado en este estudio son consistentes con algunos de las concepciones erróneas sobre variabilidad en situaciones estadísticas ya identificadas en la literatura. Por ejemplo, con tres de las presentadas por Landewehr y citadas por Shaughnessy (1992):

- Las personas creen inadecuadamente que no existe variabilidad en el 'mundo real',

- Las personas tienen una confianza injustificada en las muestras pequeñas,

- Las personas tienen poco respeto por las pequeñas diferencias en las muestras grandes. (p. 478).

No obstante, en este estudio se observa que tales concepciones tienen un desarrollo hacia niveles de razonamiento más alto que se caracterizan por la percepción de la variabilidad y por el reconocimiento del papel del tamaño de la muestra (Lee, Angotti \& Tarr, 2010).

Con relación al desarrollo de los niveles de razonamiento sobre la aleatoriedad, resulta fundamental la adquisición y uso de un lenguaje probabilista (Gal, 2005; Makar \& Rubin, 2009) que incluya un uso racional de expresiones como "no se puede saber", "es igualmente probable", "es probable", etc., y la aceptación de la existencia de una probabilidad explícita o subyacente que pueda ser aproximada mediante las frecuencias relativas (Konold, et al., 2011). De ahí la importancia de utilizar en la enseñanza situaciones en las que se aplica la definición clásica, pues esta proporciona una probabilidad a priori para compararla con las frecuencias relativas que resultan de repetir el experimento muchas veces. Aunque esto no es suficiente, como se ha puesto 
en evidencia con relación a la independencia, es condición necesaria para el desarrollo de un razonamiento probabilístico.

El problema que hace que los estudiantes violen la independencia es que creen que la información acerca de lo ocurrido en algunos o muchos experimentos influye necesariamente en los resultados futuros; por ejemplo, "si han ocurrido más bolas negras, se espera que la próxima sea negra" o "si han ocurrido más bolas negras, se espera que la próxima sea blanca"; estas inferencias han sido llamadas efectos de recencia positiva y negativa (Fischbein, 1975) respectivamente. No obstante, en el presente estudio las preguntas en las que surgieron estas respuestas están precedidas de un modelo de la situación que habría permitido deducir la independencia a partir del análisis del mecanismo del experimento (las bolas no tienen memoria), no obstante, se ignora éste y se sobrevalora la información de los resultados de experiencias realizadas.

Las anteriores observaciones han surgido a partir de preguntas simples en las que sólo se deben articular adecuadamente la definición clásica y el acercamiento frecuencial a la probabilidad; no obstante, para lograr dicha articulación se requiere un cierto nivel de razonamiento sobre las ideas fundamentales. La forma matemática de dicha articulación es la Ley de los Grandes Números (Feller, 1950, p. 243) cuya formulación incluye claramente enunciados relacionados con la aleatoriedad, independencia y variabilidad. En consecuencia, para que los estudiantes puedan hacer inferencias interesantes y resolver problemas a partir de las definiciones de probabilidad (clásica y frecuencial) conviene diseñar estrategias de enseñanza para desarrollar paralelamente al dominio del cálculo correspondiente, los niveles de razonamiento de los estudiantes acerca de y con las ideas fundamentales.

\section{Referencias}

Batanero, C. (2016). Understanding randomness: challenges for research and teaching. En K. Krainer y Nad'a Vondrová (Eds.), Proceedings of the Ninth Congress of European Research in Mathematics Education, CERME 9 (pp. 34-49). Praga: ERME.

Batanero, C., Arteaga, P., Serrano, L., \& Ruiz, B. (2014). Prospective primary school teachers' perception of randomness. En E. J. Chernoff \& B. Sriraman (Eds.), Probabilistic thinking; Presenting plural perspectives (pp. 345-366). Dordrecht: Springer.

Batanero, C., Green, D., \& Serrano, L. (1998). Randomness, its meanings and educational implications. International Journal of Mathematical Education in Science and Technology, 29(1), 113-123.

Batanero, C., \& Serrano, L. (1999). The meaning of randomness for secondary school students. Journal for Research in Mathematics Education, 30(5), 558-567.

Cañizares, M. (1997). Influencia del razonamiento proporcional y combinatorio y de creencias subjetivas en las intuiciones probabilísticas primarias. Tesis Doctoral. Universidad de Granada.

Carnap, R. (1945). The two concepts of probability: The problem of probability. Philosophy and Phenomenological Research, 5(4), 513-532.

Chernoff, E. (2009). Sample space partitions: an investigative lens. Journal of Mathematical Behavior, 28, 19-29.

Falk, R. (1979). Revision of probabilities and the time axis. Proceedings of the Third International Conference for the psychology of Mathematics Education (pp. 64-66). Warwick, UK: PME group. 
Feller, W. (1950). An introduction to probability theory and its applications. New York: John Willey and Sons.

Fischbein, E. (1975). The intuitive sources of probabilistic thinking in children. Dordrecht: Reidel.

Gal, I. (2005). Towards "probability literacy" for all citizens: building blocks and instructional dilemmas. En G. A. Jones (Ed.). Exploring probability in school: Challenges for teaching and learning (pp. 39-63). Nueva York: Springer.

Green, D. (1993). Data analysis: What research do we need? En L. Pereira-Mendoza (Ed.), Introducing data analysis in the schools: Who should teach it? (pp. 219-239). Voorburg, The Netherlands: International Statistical Institute.

Heitele, D. (1975). An epistemological view on fundamental stochastic ideas. Educational Studies in Mathematics, 6, 187-205.

Ireland, S., \& Watson, J. (2009). Building a connection between experimental and theoretical aspects of probability. International Electronic Journal of Mathematics Education, 4(3), 339-370.

Jabareen, J. (2009). Building a conceptual framework: Philosophy, definitions, and procedure. International Journal of Qualitative Methods, 8(4), 49-62.

Jones, G., Langrall, C., \& Mooney, E. (2007). Research in probability: Responding to classroom realties. En F. K. Lester (Ed.), Second handbook of research on mathematics teaching and learning (pp. 909-955). Nueva York: Macmillan.

Kahneman, D., \& Tversky, A. (1972). Subjective probability: A judgment of representativeness. Cognitive Psychology, 3, 430-454.

Kelly, I., \& Zwiers, F (1986). Mutually exclusive and Independence: Unravelling basic misconceptions in probability theory. En Davidson \& J. Swift (Eds.) Proceedings of the Second International Congress on Teaching Statistics (96-100). Victoria, Canada.

Konold, C. (1989). An outbreak of belief in independence? En C. Maher, G. Goldin \& B. Davis (Eds.), Proceedings of the 11th Annual Meeting of the North American Chapter of the International Group for the Psychology of Mathematics Education, (Vol. 2, pp. 203209). Rutgers NJ: Rutgers University Press.

Konold, C., Madden, S., Pollatsek, A., Pfannkuch, M., Wild, C., Ziedins, I., Finzer, W., Horton, N., \& Kazak, S. (2011). Conceptual challenges in coordinating theoretical and data-centered estimates of probability. Mathematical Thinking and Learning, 13, 68-86.

Konold, C., Pollatsek, A., Well, A., Lohmeier, J., \& Lipson, A. (1993). Inconsistencies in students' reasoning about probability. Journal for Research in Mathematics Education, 24(5), 392-414.

Lecoutre, M., Rovira, K., Lecoutre, B., \& Poitevineau, J. (2006). People's intuitions about randomness and probability: An empirical study. Statistics Education Research Journal, $5(1), 20-35$.

Lee, H., Angotti, R., \& Tarr, J. (2010). Making comparisons between observed data and expected outcomes: Students' informal hypothesis testing with probability simulation tools. Statistics Education Research Journal, 9(1), 68-96.

Makar, K., \& Rubin, A. (2009). A framework for thinking about informal statistical inference. Statistics Education Research Journal, 8(1), 82-105.

Metz, K. (1998). Emergent understanding and attribution of randomness: comparative analysis of the reasoning of primary grade children and undergraduates. Cognition and Instruction, $16(3), 285-365$. 
Moore, D. (1990). Uncertainty. En L. A. Steen (Ed.), On the shoulders of giants (pp. 95-138). Washington D. C.: National Research Council.

Piaget, J., \& Inhelder, B. (1975). The origin of the idea of chance in children. Nueva York: W.W. Norton.

Prodromou, T. (2012). Connecting experimental probability and theoretical probability. ZDM Mathematics Education, 44, 855 - 868.

Sánchez, E., García, J., \& Medina, M. (2014). Niveles de razonamiento y abstracción de estudiantes de secundaria y bachillerato en una situación-problema de probabilidad. Avances de Investigación en Educación Matemática, 6, 5-23.

Shaughnessy, J. (1992). Research in probability and statistics: Reflections and directions. En D. A. Grows (Ed.), Handbook of research on mathematics teaching and learning (pp. 465-494). Reston, Va: National Council of Teachers of Mathematics.

Shaughnessy, J. (1997). Missed opportunities in research on the teaching and learning of data and chance. En F. Biddulph \& K. Carr (Eds.), Proceedings of the $20^{\text {th }}$ Annual Conference of the Mathematics Education Research Group of Australasia (pp. 6-22). Rotorua, Nueva Zelanda: University of Waikato.

Shaughnessy, J., Watson, J., Moritz, J., \& Reading, C. (1999). School mathematics students' acknowledgment of statistical variation. Paper presented at the Research Pre-session of the 77th Annual meeting of the National Council of Teachers of Mathematics. San Francisco, CA: NCTM.

Stohl, H., \& Tarr, J. E. (2002). Developing notions of inference using probability simulation tools. Journal of Mathematical Behavior, 21, 319-337.

Truran, K., \& Truran, J. (1999). Are dice independent? Some responses from children and adults. En Zaslavsky O. (Ed.), Proceedings of the 23rd Conference of the International Group for the Psychology of Mathematics Instruction, (Vol. 4, pp. 289-296). Haifa: Israeli Institute of Technology.

Watkins, A. (1993). Students can compute, but can they reason? Research and Teaching in Developmental Education, 10(1), 85-94.

Watson, J., \& Kelly, B. (2004). Expectation versus variation: Students' decision making in a chance environment. Canadian Journal of Science, Mathematics and Technology Education, 4(3), 371-396.

Watson, J., Kelly, B., Callingham, R., \& Shaughnessy, M. (2003). The measurement of school students' understanding of statistical variation. International Journal of Mathematical Education in Science and Technology, 34(1), 1-29.

\section{Referencias de los autores}

Ernesto Sánchez ${ }^{1}$, CINVESTAV-IPN (México). esanchez@cinvestav.mx

Julio C. Valdez², CINVESTAV-IPN (México).jvaldez@cinvestav.mx 


\title{
The fundamental ideas of probability in the reasoning of high-school students
}

\author{
Ernesto Sánchez ${ }^{1}$, CINVESTAV-IPN (México) \\ Julio C. Valdez², CINVESTAV-IPN (México)
}

This study is located within a line of research that seeks to develop probabilistic reasoning that could promote a better performance of students in situations involving uncertainty. In order to achieve this goal, we analyze the reasoning of 30 high school students (ages 17-18), who had previously taken the course of Statistics and Probability that is offered in this level in their institution, when responding to three versions of a questionnaire. Each questionnaire deals with the comparison of probabilities in an urn context and the questions demand conceptual reasoning and not only probability calculation. Specifically, we observe the inferences the students are able to formulate from their knowledge of the classical and frequentist approaches; the most adequate setting to observe the inferences they make is seeing how they articulate these approaches.

The analysis of the responses reveals the students' tendency towards probability calculation based, most of the time, on spurious reasoning involving the notions of variability, randomness and independence. Thus, there is a number of difficulties the students often face to provide adequate responses: the overestimation of the results in the samples; an inadequate handling of random variability; the inability to recognize uncertainty once a numeric value is given to an event; an inadequate knowledge of the Law of Large Numbers (LLN); failing to apply independence when it is suitable; and a poorly developed probability concept. In addition, we observe that making probabilistic situations more experiential hardly allows the students to overcome such difficulties; therefore, the teacher's guidance becomes necessary. Using the adequate tasks and appropriate questions, the teacher can turn the simulation into a bridge between the students' ideas and the corresponding normative concepts.

As the main result, we present a hierarchy including the four developmental levels of probabilistic reasoning on the notions of variability, randomness and independence which was elaborated from the students' answers. The lowest level is characterized by the absence of this reasoning, while the highest, by the proficient use of the appropriate reasoning that students are considered to be able to formalize in this stage. This result is the basis of our suggestion that the first objective of probability teaching be the development of reasoning on these notions before or at the same time as the mathematics involved in order to set a solid ground for the students' development of formal probabilistic reasoning. 